\title{
Detection of immune-related cells and genes in the glioma tumor microenvironment from the CGGA database using bioinformatics strategies
}

Xudong Liu

Xinyang Normal University

JinYue Yin

Xinyang Normal University

Zhe Liu

City University of Hong Kong

Mengdi Fan

Xinyang Normal University

Ying Ma

Xinyang Normal University

Yu Zhang

Xinyang Normal University

Tao Peng

Xinyang Normal University

Lijun Zhao

Xinyang Normal University

Jinke Fan

Xinyang Normal University

Yafan Wang

Xinyang Normal University

Rui Li

Xinyang Normal University

Xue Zhao

Xinyang Normal University

Jiahuan Cai

Xinyang Normal University

Xinqiang Song

Xinyang Normal University

\section{Yuxuan Chen}

People's Liberation Army

Lei Wang ( $\nabla$ wangleibio@126.com ) 


\section{Research Article}

Keywords: Glioma, Tumor microenvironment, Immunotherapy, CGGA, Prognosis

Posted Date: February 9th, 2022

DOI: https://doi.org/10.21203/rs.3.rs-1327378/v1

License: (9) This work is licensed under a Creative Commons Attribution 4.0 International License. Read Full License 


\section{Abstract}

Background: Glioma is one of the most common and aggressive types of primary malignant tumors in the neuroectoderm, with high mortality rates, and recurrence. Immune checkpoint inhibitors have provided new insights in the treatment of tumors. Yet, there are still a lack of effective immune markers and therapeutic targets for glioma immunotherapy.

Material and Methods: First, The data of glioma and corresponding clinical characteristics obtain from the CGGA database. Secondly, we calculated the abundance of immune cells for each sample by the CIBERSORT algorithm and then identified immune cells associated with survival by Kaplan-Meier survival analysis. Critical immune genes were identified naturally by differential gene analysis, functional enrichment analysis, protein interaction analysis, and co-expression analysis. Eventually, the TIMER database was used to analyze further the level of immune infiltration of key immune cells.

Results: Here, we have discovered macrophages $\mathrm{M0}$ and monocytes, are associated with the survival of glioma, and three immune genes associated with survival were also uncovered, of which BIRC5 is an immune gene-specific to monocytes. The BUB1B and NCAPH are immune genes specific to macrophages M0. Furthermore, three immune genes were positively correlated with the infiltration levels of immune cells such as purity and dendritic cell and had a high response to anti-PD-1 treatment.

Conclusions: In conclusion, we have identified macrophages M0 and monocytes, and three specific immune genes that may be of high research value in glioma immunotherapy. Our work may provide new research insight into the diagnosis and prognosis of glioma.

\section{Introduction}

Glioma is a primary intracranial cancer that is the most common and predominantly aggressive [1]. It appears to be a malignant tumor of several cellular neoplasm origins with neural stem cell-like characteristics and is characterized by high mortality and recurrence [2]. Radiotherapy after surgery combined with temozolomide is currently the main treatment for patients with glioma[3]. Nevertheless, the prognosis of glioma remains disappointing, with an average 5-year survival rate of $4.7 \%$ [4], and median overall survival (OS) of 14.6 months. In 2021, the World Health Organization (WHO) classification of central nervous system (CNS) tumors redefines glioma classification by adding histologic classification, grade, and molecular genetics. It introduces new tumor types and subtypes that provide a basis for better understanding the prognosis and optimally treating patients with specific CNS cancers [5]. To date, it remains that no effective treatment or diagnosis of glioma is available.

With the accelerated advancement of next-generation sequencing technology, the volume of bioinformatics data has exposed an explosive growth [6]. Great progress has been made in medical research, especially in tumor research [7]. Recently, tumor immunology has rapidly developed, while immune checkpoint inhibitors are also practiced in many tumor treatments [8], and the improvement of diagnosis and treatment of glioma is promising. Immunotherapy and clinical trials targeting glioma are 
also conducted on a large scale, but whose outcome is not remarkable. Since tumor immune escape is universal, tumor immunotherapy can also be mainly used to personalize diagnostic treatment for patients. To date, immunotherapy for glioma remains highly challenging, and prediction of immunotherapeutic biomarkers and the search for new effective therapeutic targets have been a critical concern.

The tumor microenvironment (TME) is the field that tumor cells interact with immune cells and immunoregulatory substances in the immune system [9]. Various immune cell subsets are recruited into the tumor microenvironment through recognition of chemokines and chemokine receptors and play a remarkably essential role in tumorigenesis [10]. They also influence the diagnosis and treatment of tumors [11]. Immune evasion ability is expected to provide novel insight into cancer diagnosis and treatment as a new feature to utilize cancer cells. Immune checkpoint inhibitors, represented with antiCTLA-4 and anti-PD antibodies, and CAR-T, considered a form of secondary immune cell therapy, play an essential part in anti-tumor treatment [12]. TME plays an influential role in the fight against tumors and immune cells and provides an opportunity for the immune escape of tumor cells [13]. Therefore, the effect of immune elements in TME on the expression of tumor cell genes and the effect of clinical drugs can also influence the overall therapeutic outcome of tumors.

Cancer immunotherapy mainly involves the mutual recognition between immune substances in the tumor microenvironment and tumor cells to play a role in removing cancer cells by stimulating and enhancing the antibody immune response of the body [14]. Currently, targeting immunotherapy such as CTLA-4, PD1 , and PDL1 is the immunotherapy of tumors that has achieved great achievements [15]. In the current report, Two survival-associated immune cells and three immune cell-specific prognosis-associated genes were identified and key immune cells and biomarkers may serve as new immunotherapeutic targets for glioma.

\section{Materials And Methods}

\section{Data capture and advanced processing}

RNA-Seq gene expression profiles of glioma patients were first downloaded from the Chinese Glioma Genome Atlas (CGGA, http://www.cgga.org.cn/) database. The clinical data, mainly on gender, age, tumor grade, clinical stage, and survival time, were also downloaded.

\section{The identification of immune cells with prognostic significance}

The CIBERSORT is a tool for deconvolution of expression matrices of human immune cell subtypes using principles from linear support vector regression [16]. The method is based on a known reference set of gene expression features for 22 immune cell subtypes. The calculation of immune cell abundance was performed as follows. 
$m=f \times B(1)$

m: represents a vector composed of mixtures GEP (input requirement).

f: represents a vector composed of the proportion of the signature for each of the types of cells (unknown)

B: represents a "signature matrix" composed of the signature genes for the subset of the cells of interest (input requirement)

In the present study, we ran CIBERSORT in local $\mathrm{R}$ software to calculate the abundance ratio matrix of immune cells in gliomas. $\mathrm{P} \leq 0.05$ was used as a criterion for filtering out samples. The correlation analysis was then performed on the abundance of immune cells in the filtered samples. At the same time, Kaplan Meier analysis of the overall survival of immune cells in 22 was performed based on their abundance, with the median as the cut-off level, and tested with Log-Rank. Then, immune cells associated with survival were identification.

\section{The recognition of candidate immune cells in association with clinical information}

The abundance of candidate immune cells was analyzed in the 159 samples with the WHO classification, age ( $>45$ and $<45$ ), and IDH mutation, in combination with immune cell abundance and clinical characteristics. To explore the relationship between candidate immune cells and clinical, independent samples t-test was used for two variables, while an one-way ANOVA test was used for variables with item variables $>2$.

\section{The identification of immune-related genes}

Candidate immune cells were selected and classified into high and low groups according to their median. To obtain the differentially expressed genes of the candidate immune cells, we used the Limma $R$ language package for performing differential expression gene analysis. Here, criteria for differential analysis were set to the condition of $|\log \mathrm{FC}|>0.8$ and $\mathrm{P}<0.05$.

\section{Functional annotation of immune-related genes}

The Database for Annotation, Visualization, and Integrated Discovery [17] (DAVID, https://david.ncifcrf.gov/) and Metascape (http://metascape.org/gp/) are the most frequently used tools for $\mathrm{GO}$ term analysis and classification and gene enrichment analysis, respectively. GO terms and KEGG pathways were identified by biological process (BP), molecular function (MF), and cellular component (CC). $P<0.05$ was used as a criterion for screening. 


$$
P=1-\sum_{I=0}^{m-1} \frac{\left(\begin{array}{c}
M \\
i
\end{array}\right)\left(\begin{array}{c}
N-M \\
n-i
\end{array}\right)}{\left(\begin{array}{c}
N \\
n
\end{array}\right)}
$$

2

$\mathrm{N}$ represents the number of all genes in Go annotations; $\mathrm{n}$ is the number of differentially expressed genes;

$M$ is the number of all genes annotated for a specific $\mathrm{GO}$ term;

$\mathrm{M}$ is the $\mathrm{GO}$ term annotation for a certain number of differentially expressed genes.

The calculated p-values were Bonferroni-corrected to a p-value of 0.05 threshold, and eligible GO terms were defined as $\mathrm{GO}$ terms significantly enriched in differentially expressed genes, and the main biological functions of exercising differentially expressed genes could be determined by GO function significant enrichment analysis.

\section{The construction of PPI network and identification of hub genes}

To better understand protein-protein interactions in immune-related genes, a PPI interaction network was established using the STRING web server [18] (https://cn.string-db.org/). PPI node pairs with a combined score $\geq 0.4$ were selected for further analysis. The cytoHubba plugin [19] in Cytoscape software was used to identify the genes with the highest connectivity as hub genes for identification.

$$
\operatorname{MCC}(v)=\sum_{C \in S(v)}(\mid C-1) !
$$

3

where $S(v)$ is the product of all positive integers containing $v$ and $(i-1)$ ! is the product of all positive integers that are less than $\mathrm{Cl}$. If there are no edges between neighbors of node $\mathrm{v}$, then $\mathrm{MCC}(\mathrm{v})$ is equal to its degree.

\section{Detection and validation of immune prognosis-related genes}

To investigate the prognostic effect of hub genes, we combined the dataset of hub genes with clinical data and used one-way Cox proportional risk regression analysis (hazard ratio $(H R) \neq 1, p<0.05$ ) to screen for genes associated with prognosis. The training set was divided into high and low expression groups according to the expression of each gene. The survival $\mathrm{R}$ language package was used for performing Kaplan-Meier (K-M) analysis and a one-sample log-rank test to show the relationship between candidate gene expression and OS in patients with glioma. The final prognosis-related gene selection genes were obtained. 


\section{Immunological correlation analysis of hub immune-related genes}

Tumor Immunity Estimation Resource [20] (TIMER, http://timer.cistrome.org/) allows for a systematic and comprehensive analysis of immune infiltration levels of immune cells in different cancers. In our work, TIMER was applied to detect the correlation of key immune gene expression in six types of infiltrating immune cells, including B cells, CD4+ T cells, CD8+ T cells, neutrophils, macrophages, and dendritic cells in glioma. Simultaneously, the Submap algorithm was performed to predict the clinical response to PD-1 and PD-L1 immune checkpoint blockade therapy in high and low expression subgroups of key immune genes [21]. P-value $<0.05$ was statistically significant.

\section{Identification of small-molecule drugs with hub immune- related gene effect}

The CMAP database (https://portals.broadinstitute.org/CMap/) consists of 7056 gene expression profiles induced by 1309 small molecules widely used to explore the potential uncharted effects of existing drugs on the disease [22]. In this investigation, the drug development tool was exploited to mine small molecule drugs that worked with critical genes in glioma. A cutting criterion of $p<0.05$ was adopted. Enrichment scores (from - 1 to +1 ) were calculated to evaluate the similarity between genes and drugs. When enrichment scores were $>0$ were considered drugs with potential synergistic effects on glioma, indicating that they could mimic the biological state of cancer cells. On the contrary, the enrichment score $<0$ indicates a potentially antagonistic effect of small molecule drugs, which are considered to reverse the biological process of cancer cells and can be used as therapeutic agents.

\section{Statistics Analysis}

In this research, R (version: 4.1.0) software has been used for statistical tests and graph drawing. Wilcoxon test has been used for statistical differences between the two groups, and Kruskal-Wallis $\mathrm{H}$ test has been used for comparing multiple groups. Statistical significance was defined as $p<0.05$.

\section{Results}

\section{Data sources and preprocessing}

The gene expression profiles and matching clinical data of 693 glioma patients were downloaded from the China Glioma Database (Supplement Table1). The technological line of the article is shown in Supplement Figure 1.

\section{Acquiring the immune cells associated with the prognosis of glioma}

After CIBERSORT filtering by the deconvolution algorithm, we got 159 from 693 samples. The proportion of 22 immune cell types in each sample was also calculated (Figure 1A, B). Monocytes, type 2 
macrophages (M2), and mast cells activated in the TME of gliomas represent a higher proportion of all cells, while the percentage of cells such as B cells, T cells CD4 naïve, and Eosinophils is lower.

Subsequently, the Kaplan-Meier analysis was used to correlate the abundance ratios of 22 immune cell types with overall patient survival. It was discovered that the abundance ratios of monocytes $(p=0.15 \times$ $\left.10^{-3}\right)$ and macrophages MO $\left(p=0.16 \times 10^{-1}\right)$ were correlated with patient survival (Figure 1C, D).

\section{Identification of critical immune-related genes in macrophages M0}

When we identified macrophages $\mathrm{MO}$ as a critical immune cell in glioma, which we analyzed for correlation with clinical characteristics, we found is that macrophages $\mathrm{MO}$ correlates strongly with WHO mutation, methylation, and classification While M0 was not statistically correlated with codeletion, gender and primary recurrence status. (Figure 2A). Subsequently, 548 up-regulated immune-related genes and 289 down-regulated immune-related genes were identified in differential analysis of macrophages M0 sub-cell population (Figure 2B). Gene function annotation demonstrated that immune-related genes were majorly involved in positive regulation of cell proliferation, leukocyte migration, cell division, and other functions (Figure 2C, Supplement Table 2); KEGG pathway enrichment analysis revealed that immunerelated genes were mainly involved in Ras signaling pathway, PI3K-Akt signaling pathway, p53 signaling pathway, and other classical biological pathways of glioma (Figure 2D, Supplement Table 3). We performed protein-protein interaction network analysis to explore the interactive relationship of immunerelated genes and to get hub genes. The most connected network nodes were identified by the Cytoscape plugin cytoHubba, we obtained 22 hub genes, their connectivity was consistent (MCC score $=1.93 \mathrm{E}+70$ ). (Figure 2E, F).

\section{Identification of critical immune-related genes in monocytes}

The monocytes are another critical immune cell for glioma that we identified. We found a strong correlation between monocytes and WHO mutations (Figure 3A). Next, 246 up-regulated immune-related genes and 646 down-regulated immune-related genes were identified during differential analysis of monocytes (Figure 3B). Gene function annotation showed that immune-related genes mainly function with cell proliferation, mitochondrial nuclear division, inflammatory response, etc. (Figure 3C, Supplement Table 4). KEGG pathway enrichment analysis demonstrated that differential immune-related genes were mainly involved in biological pathways such as Rap1 signaling pathway, PI3K-Akt signaling pathway, etc. (Figure 3D, Supplement Table5). As similar analysis as macrophages M0 for monocytes was used to identify the 21 hub genes $(\mathrm{MCC}$ score $=5.51 \mathrm{E}+67)$ with the greatest connectivity (Figure 3E, F).

\section{Recognition immune-related genes specific to macrophages $\mathrm{MO}$ and monocytes}

To further identify immune-related genes specific to macrophages M0 and monocytes, we overlapped the identified core immune-related genes in two immune cells (Figure 4A), and there was a large overlap of 
critical immune-related genes in both classes of immune cells A total of 23 genes, 20 of which are intersected, and only 3 of which are specific to both immune cell classes. We discovered that BIRC5 is an immune-related gene specific to monocytes. BUB1B and NCAPH are crucial immune-related genes specific to macrophages $\mathrm{M} 0$. Gene function enrichment analysis showed that all these hub genes are mainly involved in cell division, mitochondrial cell cycle process, meiotic nuclear division, DNA replication, and other functions (Figure 4B, Supplement Table6). We performed a Kaplan-Meier survival analysis of immune-related genes specific to two classes of immune cells. We noted a significant correlation between these three genes (BIRC5, BUB1B, and NCAPH) and clinical signatures (Figure 4C-D).

\section{Critical immune-related genes are involved in tumor immune infiltration in glioma}

Three hub immune-related genes are all potential immunotherapeutic targets and are important for immune-related studies of glioma. Meanwhile, we examined the correlation between three immune cellspecific survival-related genes and the infiltration level of immune cells by TIMER, and the results are shown in Figure 5A. The expression levels of BIRC5 were positively correlated with the infiltration levels of purity, dendritic Cell. The expression levels of $N C A P H$ were positively correlated with the infiltration levels of purity, neutrophil. The expression levels of $N C A P H$ were positively correlated with the infiltration levels of purity, neutrophil, and dendritic cell. The expression levels of BUB1B were positively correlated with the infiltration levels of purity, CD8+ T dell, and dendritic Since PD1 and PD-L1 play an essential role in tumor immunosuppression and immunotherapy, we investigated the relationship between the expression levels of three hub immune-related genes and PD1 and PD-L1, respectively. We found that the expression levels of $N C A P H$ and BIRC5 were significantly in positive correlation with the expression of PD-L1, and the expression levels of $B U B 1 B$ were significantly correlated with the expression of PD-1 and PD-L1. These data indicate that three key immune genes can be used as accurate predictors of immune features of glioma (Figure 5B).

\section{CMAP analysis identifies small molecule drugs for glioma treatment}

To acquire small molecule drugs that interact with a key immune gene, we uploaded three key immune genes to the CMAP website. Overall, eight small molecule drugs could be potential drugs for the treatment of glioma patients by shear criteria.

\section{Discussion}

Glioma is a malignant tumor of the central nervous system with a high mortality rate and a high risk of recurrence [23]. The treatment for glioma includes surgical resection, adjuvant radiotherapy, chemotherapy combined with temozolomide, etc. [24]. Nevertheless, the lack of precise understanding of the pathogenesis of glioma has resulted in a low survival rate. Recently, new therapeutic approach immune checkpoints have been discovered, which provide new ideas for immunotherapy of tumors by specifically blocking immunosuppressive effects [25]. In the present work, we screened and identified 
cells and genes significantly associated with immune infiltration and clinical features in the glioma microenvironment by bioinformatics approach. This research provides a new idea for immunotherapy of glioma.

In the present research, we discovered that two types of immune cells, macrophages $\mathrm{M} 0$ and monocytes, are closely associated with the survival of patients with glioma. During immune editing, immune cells have the function of withdrawing and evoking tumorigenic responses [26]. Macrophages are the most abundant cells in the tumor microenvironment and are highly plastic, making them perform multiple functions in the tumor microenvironment [27]. Macrophages are commonly defined as two subtypes activated and inactivated, where activated macrophages include classically activated M1 cells and alternatively activated $\mathrm{M} 2$ cells [28]. The other subtype is an inactivated macrophage called $\mathrm{M} 0$, and this type of cell typically is neither stimulated nor receives signals that promote activation and functional polarization [29]. Generally, the macrophages $\mathrm{MO}$ can be reprogrammed to polarized macrophages once the macrophages $\mathrm{M} 0$ receive $\mathrm{M} 1$ or $\mathrm{M} 2$ specific signals and in turn shift to an anti-inflammatory $\mathrm{M} 1$ phenotype, including radiotherapy and immune checkpoint blockade, such as anti-PD-L1/PD-1 strategies [27]. Monocytes are innate immune cells of the mononuclear phagocyte system and have an influential role in regulating cancer development and progression [30]. It effectively suppresses tumor-specific T-cell immunity by secreting tumor necrosis factor and stimulating monocytes to express PD-L1, PD-L1+ monocytes promote tumor growth in vivo [31]. In summary, monocytes and macrophages M0 are significantly associated with survival in patients with glioma and probably play an invaluable role in immune infiltration and immunotherapy.

GO functional annotation and KEGG pathway enrichment analysis of macrophages $\mathrm{M} 0$ and monocytes showed shared involvement in classical immune pathways such as PI3K-Akt signaling pathway, p53 signaling pathway, and ECM-receptor interaction. In the tumor microenvironment, immune cells interact through cell-cell communication 31063754. the PI3K-Akt signaling pathway, a classical signaling pathway in tumorigenesis, has an important role in activating numerous immune cells [32]. The PI3K-AKTmTOR signaling pathway has been reported to contribute to the development and activity of lymphocytes [33]. The mutation of the tumor suppressor p53 is critical for tumor evasion of apoptosis and senescence, and p53 dysfunction also promotes inflammation and supports tumor immune evasion, thereby serving as an immunological driver of tumorigenesis [34]. Targeting the p53 pathway in TME can be used to reverse immunosuppression, enhance the antitumor killed effect, and harness tumor-specific, persistent, and systemic antitumor immunity with minimal toxicity [34]. ECM-receptor interaction is instrumental in the process of tumor cell shedding, adhesion, degradation, motility, and proliferation [35]. It has been reported that ECM can promote epithelial-mesenchymal transition (EMT) of cancer cells in colorectal cancer [36]. The ECM can inhibit angiogenesis in glioma through endothelial-macrophage interactions and altered extracellular matrix in glioma [37]. Meanwhile, Platelet activation, Rap1 signaling pathway, inflammatory response, leukocyte migration, and other functions and signaling pathways are very tightly linked to tumor immunity $[38,39]$. 
Ultimately, we characterized three cell-specific immune-related genes, with BIRC5 being a monocytesspecific immunity gene and $B U B 1 B$ and $N C A P H$ being macrophages $\mathrm{M} 0$-specific immune genes.

Immunological associations with the 3 genes have not been reported in previous studies of gliomas. The BIRC5 (Survivin), which belongs to the family of apoptosis suppressor genes, is abundantly expressed in tumors[40]. It has been investigated that Brexpipazole can increase the sensitivity of glioma stem cells to osimertinib by reducing the expression of BIRC5 [41]. BUB1B is a mitotic spindle checkpoint molecule. It is associated with tumor cell aggressiveness and drug resistance in gliomas [42]. NCAPH is a structural component of chromosomes during mitosis [43]. High expression of NCAPH promotes the proliferation of colorectal cancer cells and increases radiation resistance, and NCAPH gene silencing stops the cell cycle at the G2/M phase, subsequently inducing apoptosis. In prostate cancer, knockdown of NCAPH decreased cell proliferation and inhibited the aggressiveness of prostate cancer cells.

In conclusion, we identified two immune cells and three specific immune genes associated with survival in glioma patients and demonstrated that the expression of three immune genes correlated with the overall survival of glioma patients in the present study. Macrophages $M 0$ and monocytes, two classes of immune cells, and three immune genes, $B I R C 5, B U B 1 B$, and $N C A P H$, can be used as prognostic glioma markers and guide immunotherapy glioma. The results of this work are mainly from bioinformatics experiments and remain to be further investigated by cell biology experiments. This study provides new and more valuable ideas to study the tumor microenvironment and immunotherapy and prognosis of glioma.

\section{Declarations}

\section{Author Declarations}

\section{Ethics approval and consent to participate}

Not applicable.

\section{Consent for publication}

Not applicable.

\section{Availability of data and materials}

Publicly available datasets were analysed in this study. The gene expression RNA-Seq of glioma from the Chinese Glioma Genome Atlas (CGGA, http://www.cgga.org.cn/).

\section{Competing interests}

The authors declare that the research was conducted in the absence of any commercial or financial relationships that could be construed as a potential conflict of interest. 
This study was supported by the National Natural Science Foundation of China (U1804179), the Henan Science and Technology Innovation Team, the Investigation on Plant Resources in Dabie Mountains and the Study and Utilization of Active Components of Special plants (2017083), Henan key scientific and technological projects (202102310190), Scientific Research Foundation of Graduate School of Xinyang Normal University(2021KYJJ53).

\section{Authors' contributions}

The study conception and design were performed by LW, YC, and XS. Data collection and analysis were performed by $X L, J Y, Y Z, Y M, Y W, R L, P T, J F, L Z, M F, X Z$, JC. The first draft of the manuscript was written by $\mathrm{XL}$ and $\mathrm{ZL}$. All authors commented on previous versions of the manuscript. All authors read and approved the final manuscript.

\section{Acknowledgements}

Not applicable.

\section{Compliance with Ethical Standards}

Not applicable.

\section{Disclosure of potential conflicts of interest}

Not applicable.

\section{Research involving Human Participants and/or Animals}

Not applicable.

\section{Informed consent}

Not applicable.

\section{References}

1. McFaline-Figueroa, J.R. and E.Q. Lee, Brain Tumors. Am J Med, 2018. 131(8): p. 874-882.

2. Bao, S., et al., Stem cell-like glioma cells promote tumor angiogenesis through vascular endothelial growth factor. Cancer Res, 2006. 66(16): p. 7843-8.

3. Brada, M., Radiotherapy in malignant glioma. Ann Oncol, 2006. 17 Suppl 10: p. x183-5.

4. Perry, J.R., et al., Short-Course Radiation plus Temozolomide in Elderly Patients with Glioblastoma. N Engl J Med, 2017. 376(11): p. 1027-1037. 
5. Louis, D.N., et al., The 2021 WHO Classification of Tumors of the Central Nervous System: a summary. Neuro Oncol, 2021. 23(8): p. 1231-1251.

6. van Dijk, E.L., et al., Ten years of next-generation sequencing technology. Trends Genet, 2014. 30(9): p. 418-26.

7. Morganti, S., et al., Next Generation Sequencing (NGS): A Revolutionary Technology in Pharmacogenomics and Personalized Medicine in Cancer. Adv Exp Med Biol, 2019. 1168: p. 9-30.

8. de Miguel, M. and E. Calvo, Clinical Challenges of Immune Checkpoint Inhibitors. Cancer Cell, 2020. 38(3): p. 326-333.

9. Gajewski, T.F., H. Schreiber, and Y.X. Fu, Innate and adaptive immune cells in the tumor microenvironment. Nat Immunol, 2013. 14(10): p. 1014-22.

10. Nagarsheth, N., M.S. Wicha, and W. Zou, Chemokines in the cancer microenvironment and their relevance in cancer immunotherapy. Nat Rev Immunol, 2017. 17(9): p. 559-572.

11. Desrichard, A., A. Snyder, and T.A. Chan, Cancer Neoantigens and Applications for Immunotherapy. Clin Cancer Res, 2016. 22(4): p. 807-12.

12. Bagchi, S., R. Yuan, and E.G. Engleman, Immune Checkpoint Inhibitors for the Treatment of Cancer: Clinical Impact and Mechanisms of Response and Resistance. Annu Rev Pathol, 2021. 16: p. 223-249.

13. Hargadon, K.M., C.E. Johnson, and C.J. Williams, Immune checkpoint blockade therapy for cancer: An overview of FDA-approved immune checkpoint inhibitors. Int Immunopharmacol, 2018. 62: p. 29-39.

14. Yang, Y., Cancer immunotherapy: harnessing the immune system to battle cancer. J Clin Invest, 2015. 125(9): p. 3335-7.

15. Qin, S., et al., Novel immune checkpoint targets: moving beyond PD-1 and CTLA-4. Mol Cancer, 2019. 18(1): p. 155.

16. Newman, A.M., et al., Robust enumeration of cell subsets from tissue expression profiles. Nat Methods, 2015. 12(5): p. 453-7.

17. Dennis, G., Jr., et al., DAVID: Database for Annotation, Visualization, and Integrated Discovery. Genome Biol, 2003. 4(5): p. P3.

18. Szklarczyk, D., et al., The STRING database in 2021: customizable protein-protein networks, and functional characterization of user-uploaded gene/measurement sets. Nucleic Acids Res, 2021. 49(D1): p. D605-D612.

19. Chin, C.H., et al., cytoHubba: identifying hub objects and sub-networks from complex interactome. BMC Syst Biol, 2014. 8 Suppl 4: p. S11. 
20. Li, T., et al., TIMER: A Web Server for Comprehensive Analysis of Tumor-Infiltrating Immune Cells. Cancer Res, 2017. 77(21): p. e108-e110.

21. Ay, F., M. Kellis, and T. Kahveci, SubMAP: aligning metabolic pathways with subnetwork mappings. J Comput Biol, 2011. 18(3): p. 219-35.

22. Yang, K., et al., CMAP: Complement Map Database. Bioinformatics, 2013. 29(14): p. 1832-3.

23. Gusyatiner, O. and M.E. Hegi, Glioma epigenetics: From subclassification to novel treatment options. Semin Cancer Biol, 2018. 51: p. 50-58.

24. Bush, N.A., S.M. Chang, and M.S. Berger, Current and future strategies for treatment of glioma. Neurosurg Rev, 2017. 40(1): p. 1-14.

25. Friedman, C.F., T.A. Proverbs-Singh, and M.A. Postow, Treatment of the Immune-Related Adverse Effects of Immune Checkpoint Inhibitors: A Review. JAMA Oncol, 2016. 2(10): p. 1346-1353.

26. Wang, X., et al., The Relationship of Sphingosine Kinase 1 With Pyroptosis Provides a New Strategy for Tumor Therapy. Front Immunol, 2020. 11: p. 574990.

27. Najafi, M., et al., Macrophage polarity in cancer: A review. J Cell Biochem, 2019. 120(3): p. 2756-2765.

28. Shapouri-Moghaddam, A., et al., Macrophage plasticity, polarization, and function in health and disease. J Cell Physiol, 2018. 233(9): p. 6425-6440.

29. Tedesco, S., et al., Convenience versus Biological Significance: Are PMA-Differentiated THP-1 Cells a Reliable Substitute for Blood-Derived Macrophages When Studying in Vitro Polarization? Front Pharmacol, 2018. 9: p. 71.

30. Olingy, C.E., H.Q. Dinh, and C.C. Hedrick, Monocyte heterogeneity and functions in cancer. J Leukoc Biol, 2019. 106(2): p. 309-322.

31. Zhang, W., et al., IL-6 promotes PD-L 1 expression in monocytes and macrophages by decreasing protein tyrosine phosphatase receptor type $O$ expression in human hepatocellular carcinoma. $\mathrm{J}$ Immunother Cancer, 2020. 8(1).

32. Balaji, S., et al., NF-kappaB signaling and its relevance to the treatment of mantle cell lymphoma. J Hematol Oncol, 2018. 11(1): p. 83.

33. Zhao, R., et al., PD-1/PD- 11 blockade rescue exhausted $C D 8+T$ cells in gastrointestinal stromal tumours via the PI3K/Akt/mTOR signalling pathway. Cell Prolif, 2019. 52(3): p. e12571.

34. Guo, G., et al., Local Activation of p53 in the Tumor Microenvironment Overcomes Immune Suppression and Enhances Antitumor Immunity. Cancer Res, 2017. 77(9): p. 2292-2305. 
35. Bao, Y., et al., Transcriptome profiling revealed multiple genes and ECM-receptor interaction pathways that may be associated with breast cancer. Cell Mol Biol Lett, 2019. 24: p. 38.

36. Chen, G., et al., Oncogenic effect of PHLDB2 is associated with epithelial-mesenchymal transition and E-cadherin regulation in colorectal cancer. Cancer Cell Int, 2019. 19: p. 184.

37. Cui, X., et al., Hacking macrophage-associated immunosuppression for regulating glioblastoma angiogenesis. Biomaterials, 2018. 161: p. 164-178.

38. Malehmir, M., et al., Platelet GPIbalpha is a mediator and potential interventional target for NASH and subsequent liver cancer. Nat Med, 2019. 25(4): p. 641-655.

39. Sarvaria, A., J.A. Madrigal, and A. Saudemont, $B$ cell regulation in cancer and anti-tumor immunity. Cell Mol Immunol, 2017. 14(8): p. 662-674.

40. Lin, T.Y., et al., BIRC5/Survivin is a novel ATG12-ATG5 conjugate interactor and an autophagy-induced DNA damage suppressor in human cancer and mouse embryonic fibroblast cells. Autophagy, 2020. 16(7): p. $1296-1313$.

41. Tang, J.H., et al., Bortezomib inhibits growth and sensitizes glioma to temozolomide (TMZ) via downregulating the FOXM1-Survivin axis. Cancer Commun (Lond), 2019. 39(1): p. 81.

42. Lee, E., et al., Sensitivity to BUB1B Inhibition Defines an Alternative Classification of Glioblastoma. Cancer Res, 2017. 77(20): p. 5518-5529.

43. Xiong, Q., et al., miR-133b targets NCAPH to promote beta-catenin degradation and reduce cancer stem cell maintenance in non-small cell lung cancer. Signal Transduct Target Ther, 2021. 6(1): p. 252.

\section{Figures}




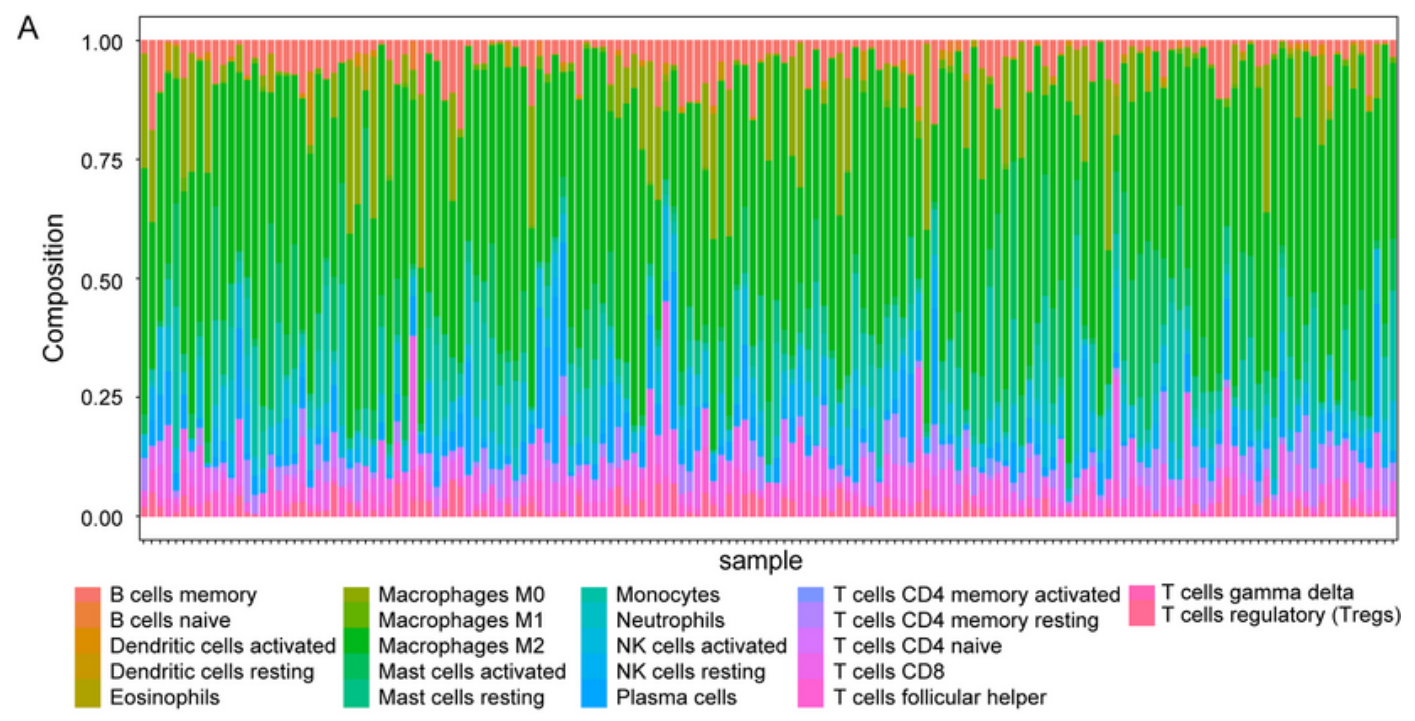

B

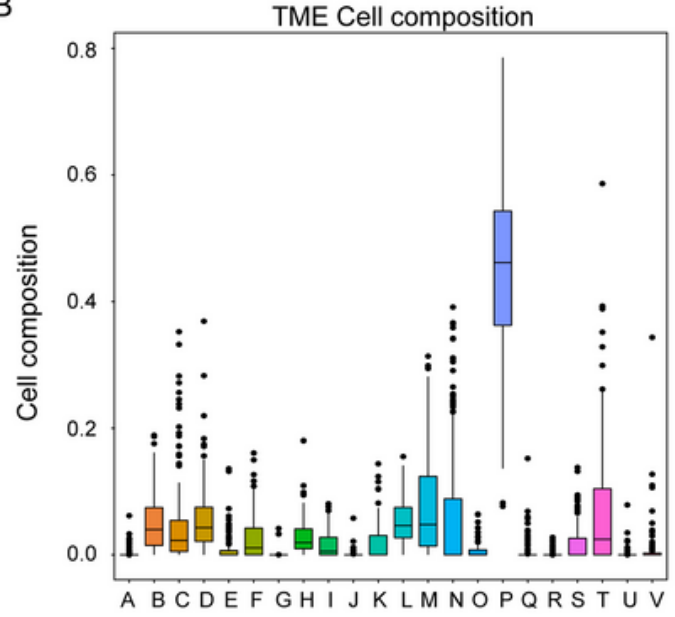

\section{CellType}

A. B cells naive

B. B cells memory

C. Plasma cells

D. T cells CD 8

E. T cells CD4 naive

F. T cells CD4 memory resting

G. T cells CD4 memory activated

H. T cells follicular helper

I. T cells regulatory (Tregs)

J. T cells gamma delta

K. NK cells resting
L. NK cells activated

M. Monocytes

N. Macrophages MO

O. Macrophages M1

P. Macrophages M2

Q. Dendritic cells resting

R. Dendritic cells activated

$\mathrm{S}$. Mast cells resting

T. Mast cells activated

U. Eosinophils

V. Neutrophils
C

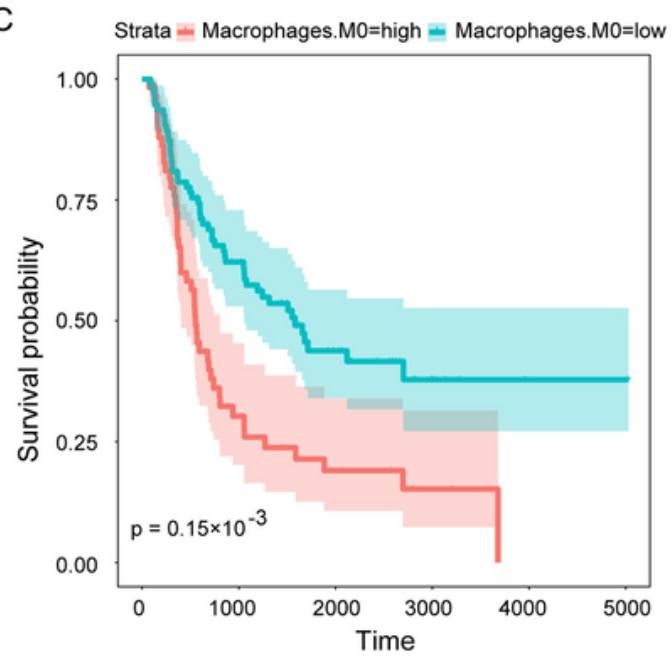

D

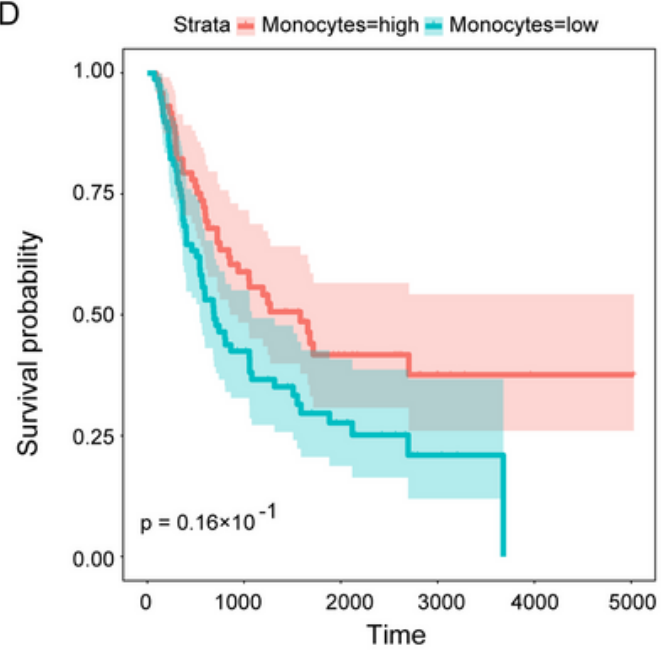

\section{Figure 1}

The identification of critical immune cells in glioma. (A-B). The histogram and the box plot demonstrate the abundance ratio of different immune cells in 163 glioma samples. The color difference indicates the immune cells of each type. (C-D). The survival analysis identified both immune cells at various abundance. Red color represents high expression, and blue color represents low expression. 

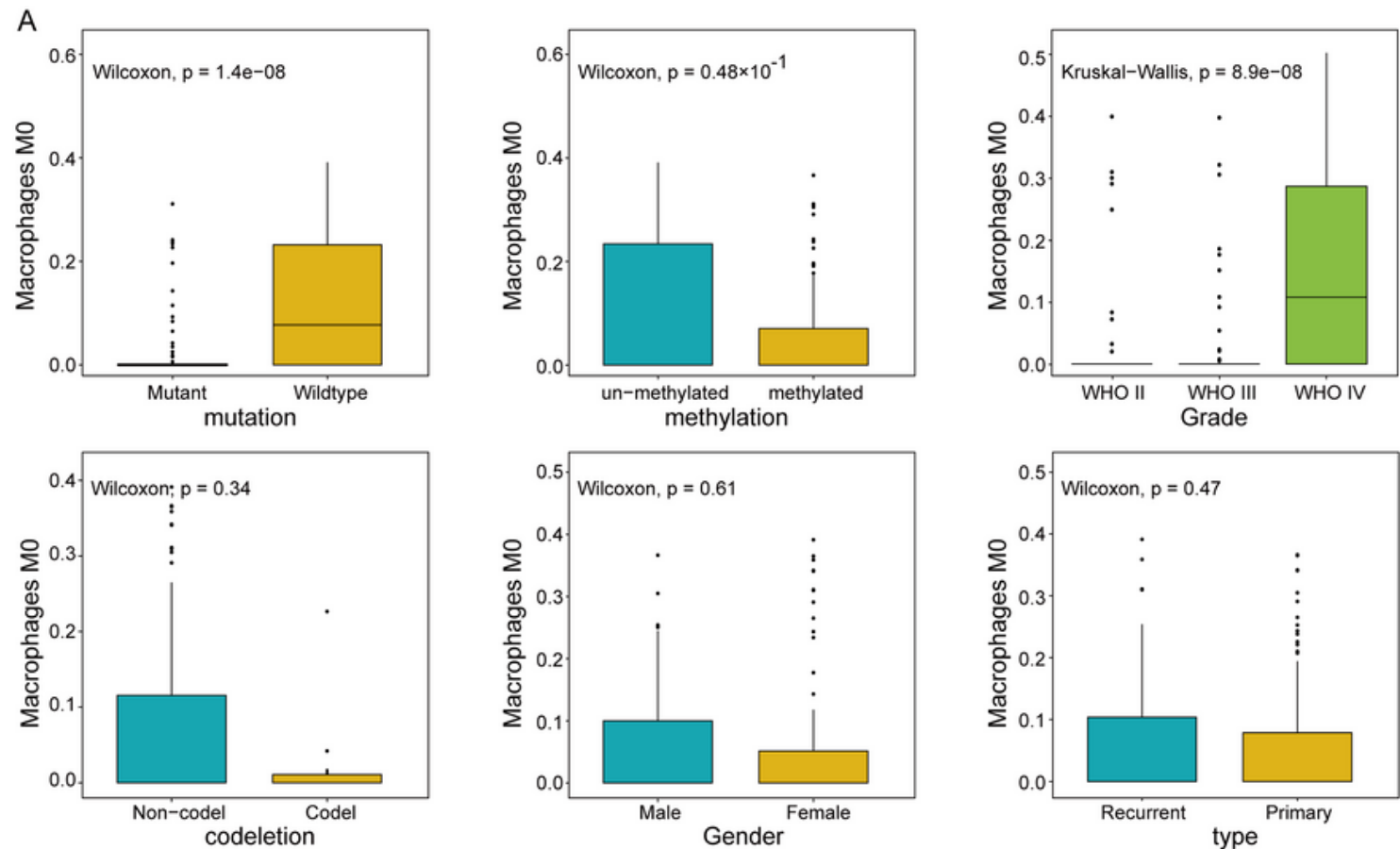

B
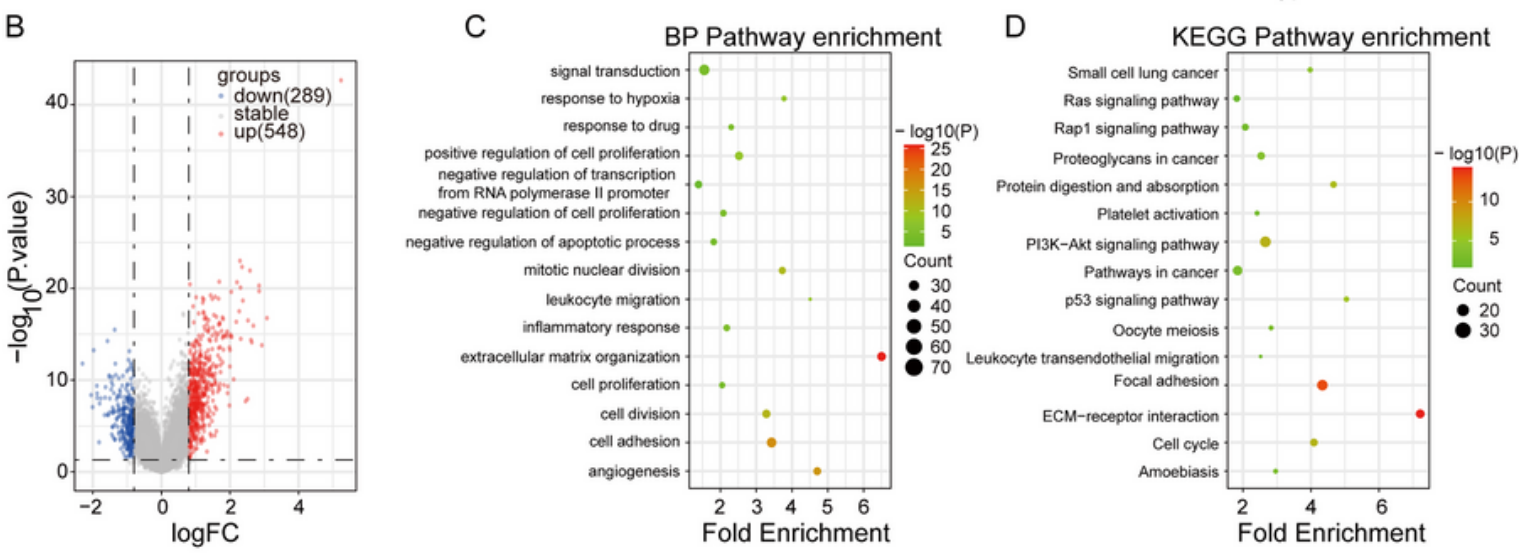

E

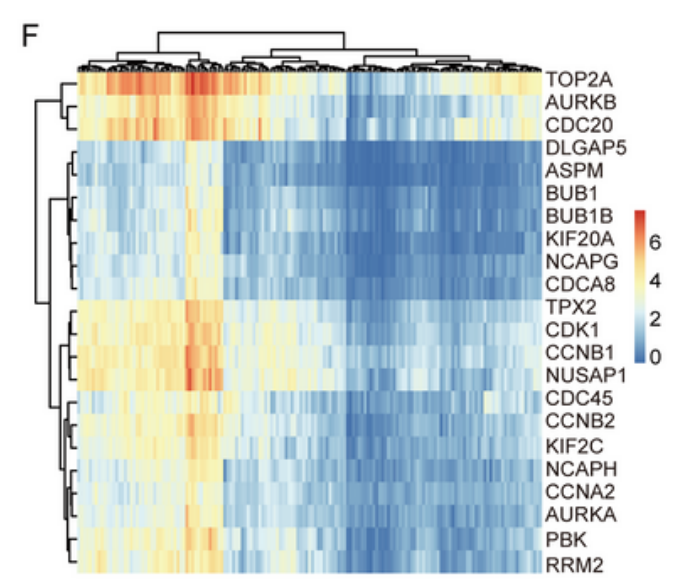

Figure 2

Clinical characteristics of monocytes and essential immune-related genes. (A). The correlation of monocytes abundance ratio with clinical features. (B). The volcano plot indicates the differentially expressed genes analyzed by clustering monocytes by their abundance. Blue color indicates downregulation; red color indicates up-regulation, and grey color indicates no significant changes. (C). GO functional annotation of immune-related genes in monocytes. (D). KEGG pathway enrichment analysis of 
immune-related genes in monocytes. (E) The PPI network of genes has been established by using cytohubba to recognize the 22 most connected hub genes. (F) The Heatmap represented the transcriptional expression profiles of 22 critical immune-related genes.
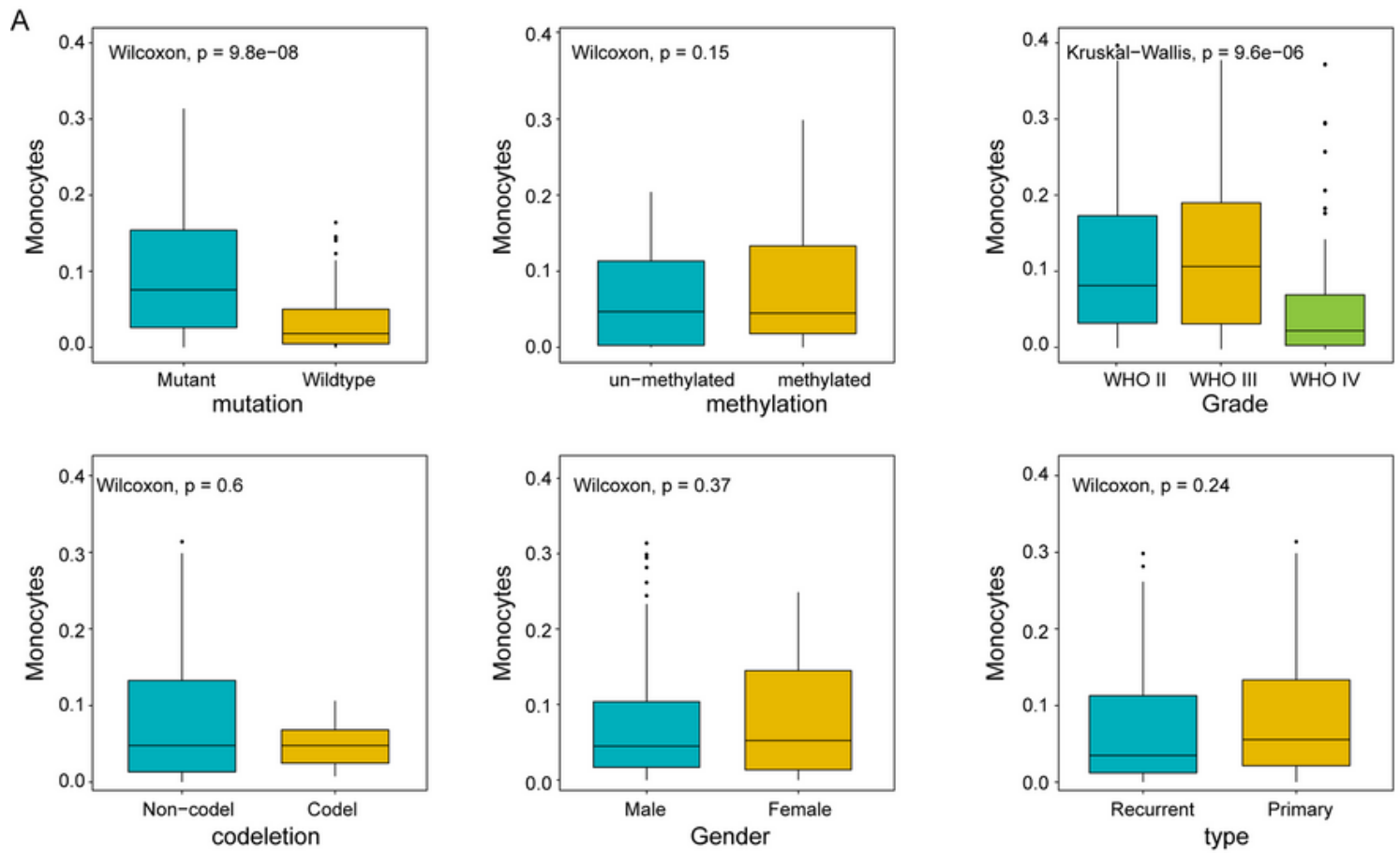

B

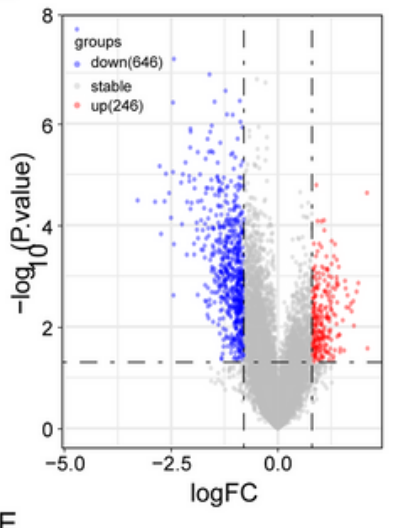

E

C

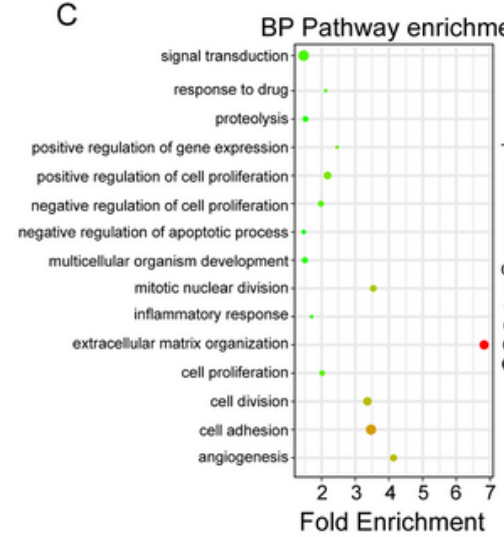

D

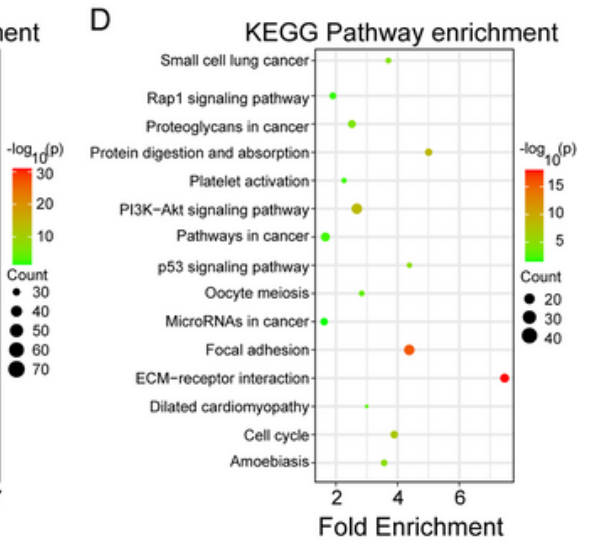

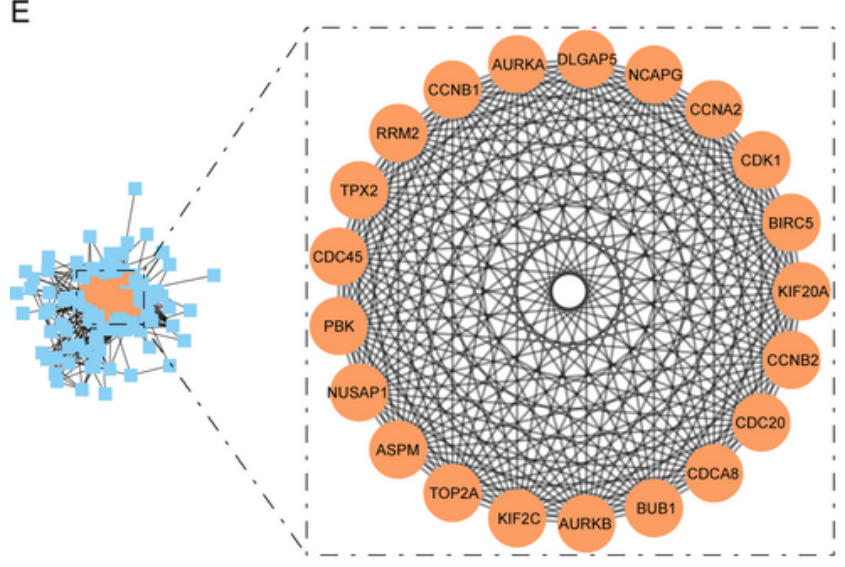

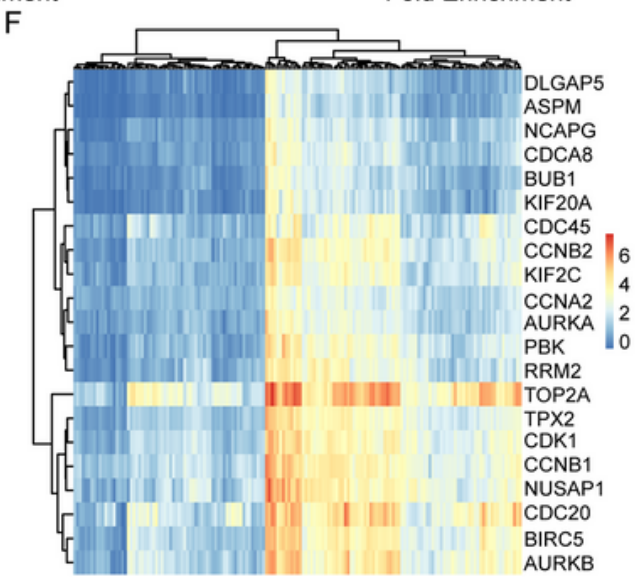

Figure 3 
Clinical characteristics of macrophages and essential immune-related genes. (A). The correlation of macrophage $\mathrm{M} 0$ abundance ratio with clinical features. (B). The volcano plot indicates the differentially expressed genes analyzed by clustering macrophage $\mathrm{MO}$ by their abundance. Blue color indicates downregulation; red color indicates up-regulation. (C). GO functional annotation of immune-related genes in macrophage MO. (D). KEGG pathway enrichment analysis of immune-related genes in macrophage M0. (E) The PPI network of genes has been established by using cytohubba to recognize the 22 most connected hub genes. (F) The Heatmap represented the transcriptional expression profiles of 22 critical immune-related genes. 
A

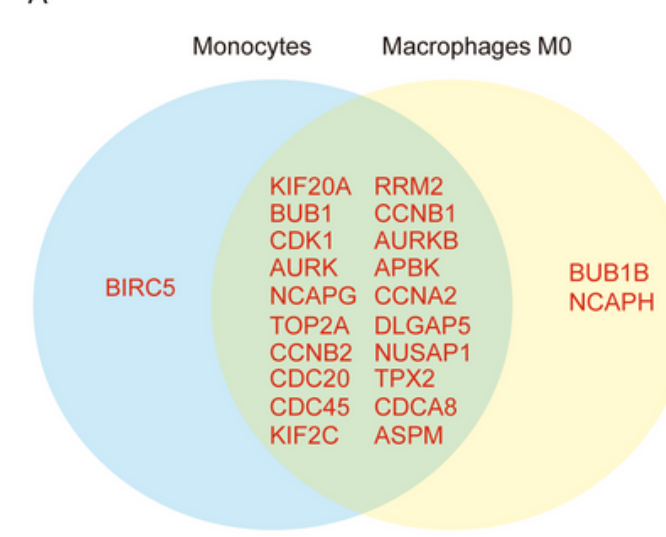

B

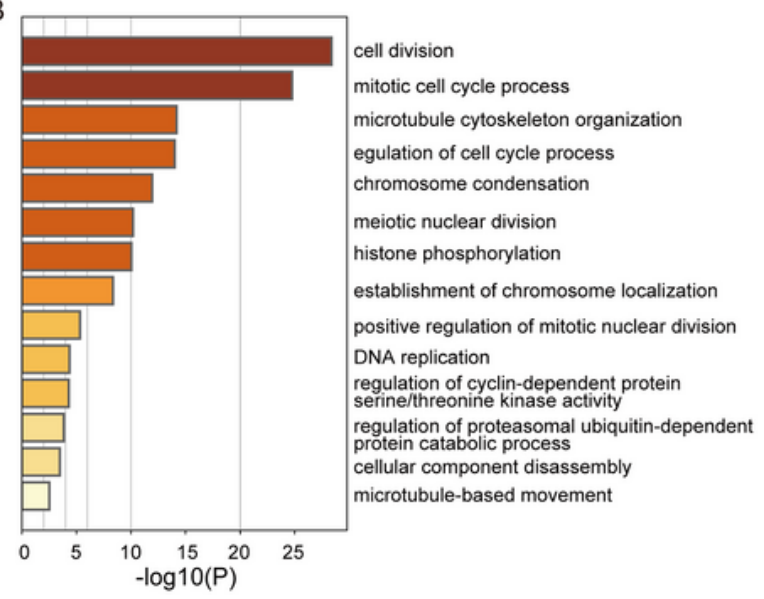

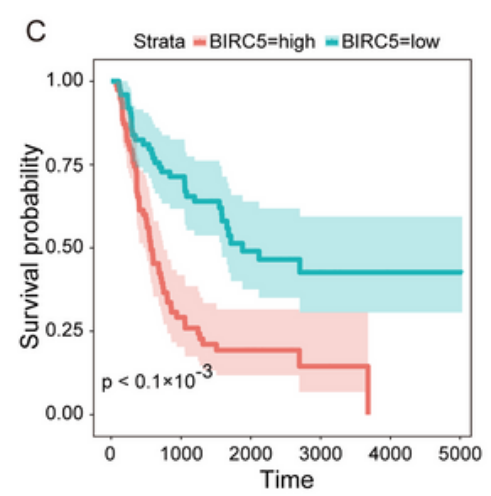
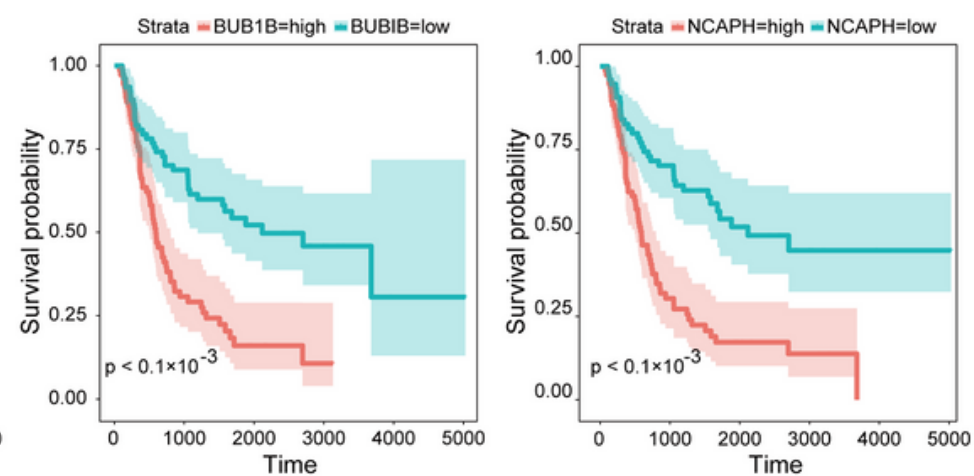

D
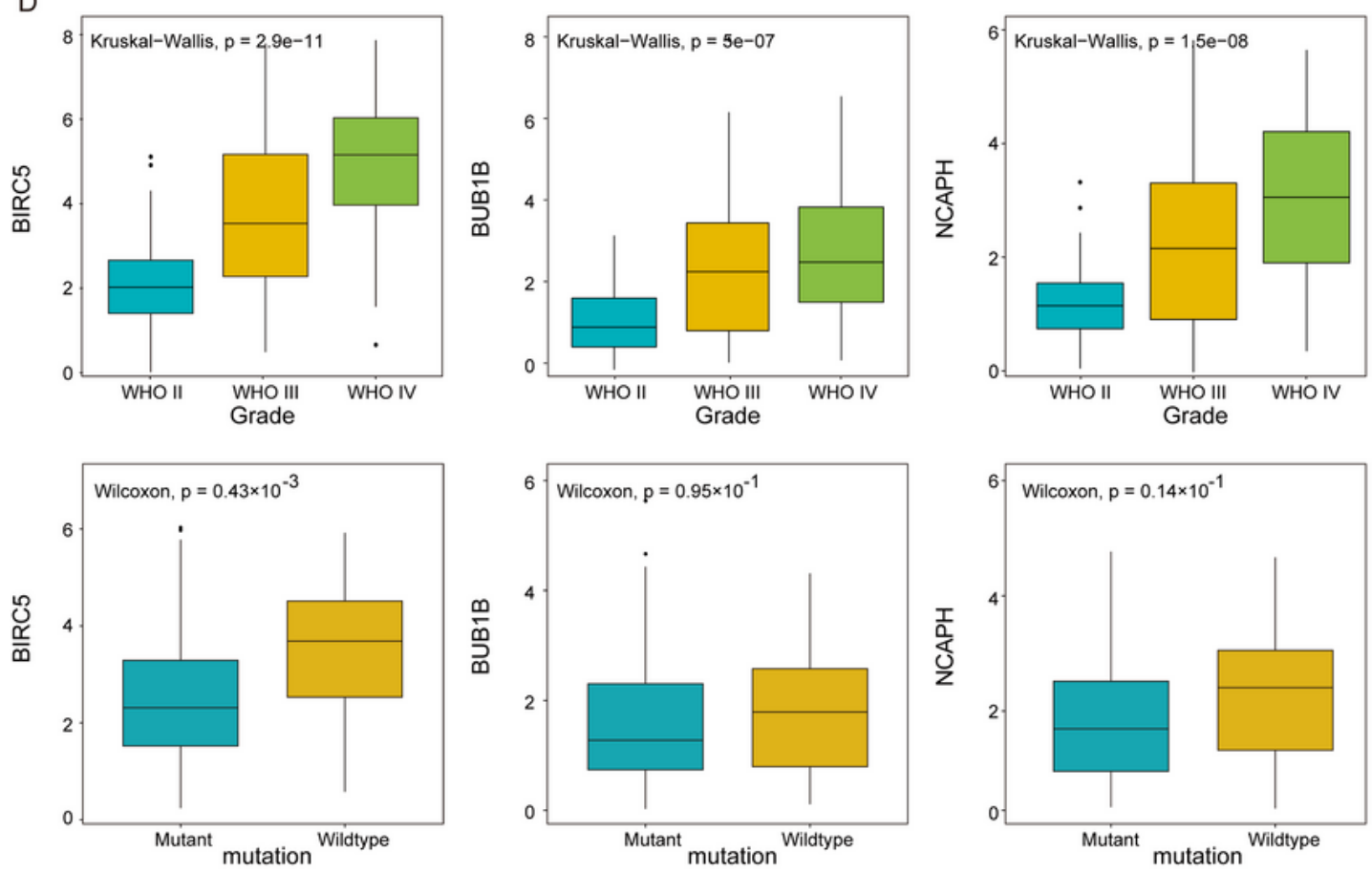

Figure 4

Immune cell-specific immune-related gene identification and correlation analysis of clinical features.

(A). Venn diagram displaying the genes of the two immune cells involved in infiltration. (B). GO functional annotation of critical immune-related genes. (C). Survival analysis of key immune-related genes. (D). Correlation analysis of critical immune-related genes with clinical features. 
A
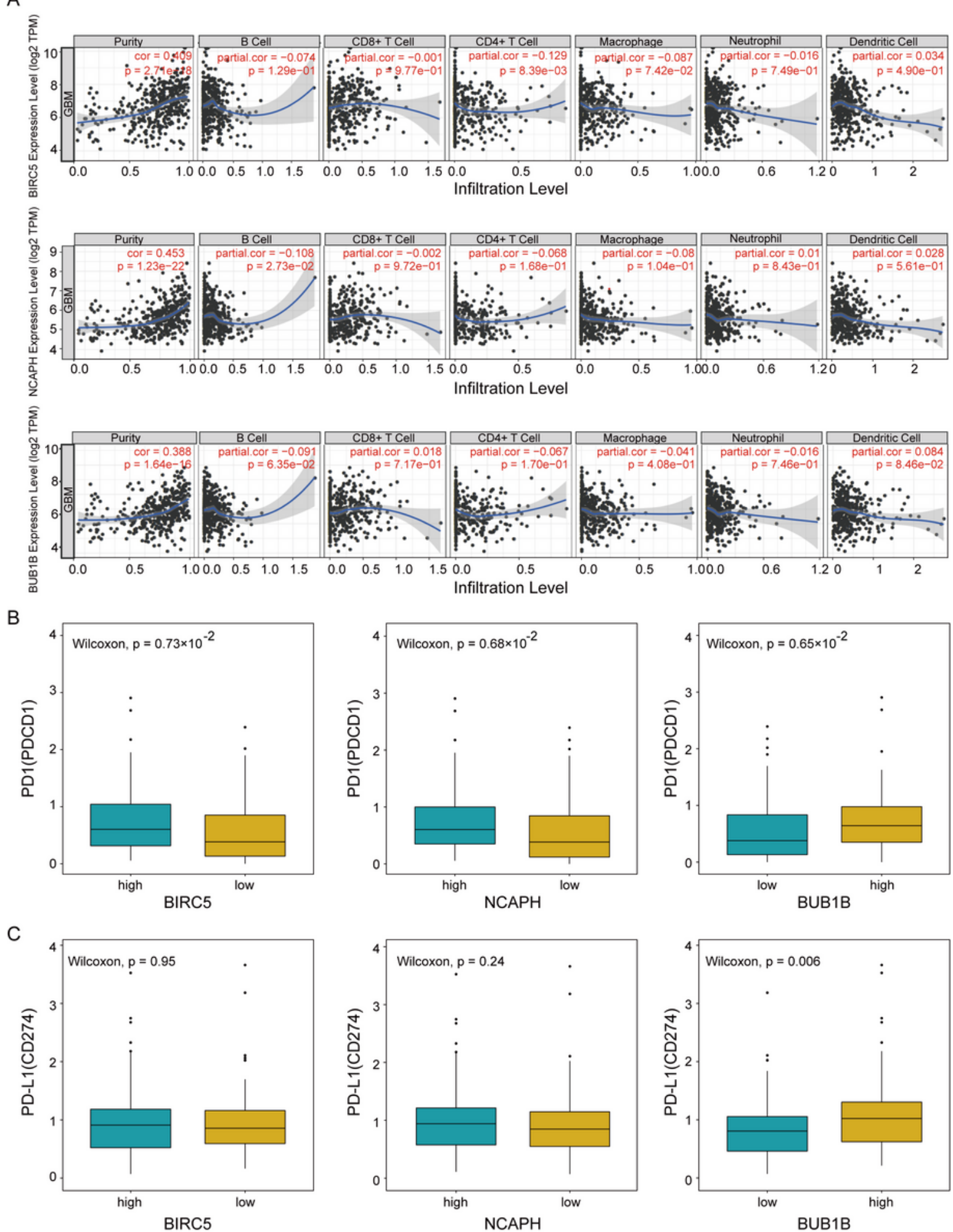

Figure 5

Immune correlations of essential immune-related genes. (A). The correlation analysis of expression levels of essential hub immune-related genes with the contents of immune cells. (B). PD1 and PD-L1 expression levels in the dataset with correlation analysis of critical immune-related genes (Wilcoxon test).

\section{Supplementary Files}


This is a list of supplementary files associated with this preprint. Click to download.

- SupplementFigure1.tif

- SupplementFigure2.tif

- SupplementTable.docx 